\title{
Nonlinear eigenvalue problems for coupled Helmholtz equations modeling gradient-index graphene waveguides
}

\author{
Jung Heon Song ${ }^{\mathrm{a}, 1,2}$, Matthias Maier ${ }^{\mathrm{b}, 1,3, *}$, Mitchell Luskin ${ }^{\mathrm{a}, 1,2}$ \\ a School of Mathematics, University of Minnesota, 206 Church Street SE, Minneapolis, \\ $M N$ 55455, USA. \\ ${ }^{b}$ Department of Mathematics, Texas A\&M University, 3368 TAMU, College Station, \\ $T X 77843, U S A$.
}

\begin{abstract}
We discuss a quartic eigenvalue problem arising in the context of an optical waveguiding problem involving atomically thick $2 D$ materials. The waveguide configuration we consider consists of a gradient-index (spatially dependent) dielectric equipped with conducting interior interfaces. This leads to a quartic eigenvalue problem with mixed transverse electric and transverse magnetic modes, and strongly coupled electric and magnetic fields. We derive a weak formulation of the quartic eigenvalue problem and introduce a numerical solver based on a quadratification approach in which the quartic eigenvalue problem is transformed to a spectrally equivalent companion problem. We verify our numerical framework against analytical solutions for prototypical geometries. As a practical example, we demonstrate how an improved quality factor (defined by the ratio of the real and the imaginary part of the computed eigenvalues) can be obtained for a family of gradientindex host materials with internal conducting interfaces. We outline how this result lays the groundwork for solving related shape optimization problems.
\end{abstract}

Keywords: Guided mode, time-harmonic Maxwell's equations, surface plasmon polariton, nonlinear eigenvalue problem, quartic eigenvalue problem, quadratification 2010 MSC: 65N30, 78M10, 78M30, 35P30 


\section{Introduction}

Surface plasmon polaritons (SPPs) are charge density waves that are coupled to electromagnetic (EM) waves at the interface between a metal and a dielectric substrate. Exhibiting strong confinement and relatively low propagation losses, they are thought to be a novel way to confine and control light on the subwavelength scale in the field of nanophotonic technology. Such SPPs can be excited in graphene, a two-dimensional carbon allotrope with a single atom layer that is arranged in a honeycomb lattice structure [1]. It is characterized by strong confinement, low losses, and extreme tunability [1, 2]; In the infrared regime, the electric surface conductivity of such a $2 \mathrm{D}$ material is characterized by being complex-valued with a dominant positive imaginary part. This allows for the propagation of SPPs. Plasmons on graphene offer not only a lower ohmic loss than conventional plasmonic materials, but also a strong subwavelength confinement of the EM field [3, 4]. The tunability of graphene by electrical gating or chemical doping, makes graphene a promising candidate for future compact plasmon devices [5].

A conventional approach of analyzing a waveguide problem is to first reduce Maxwell's equations to a Helmholtz eigenvalue problem. For a homogeneously filled waveguide, the EM fields decouple from one another, making the numerical simulation straightforward. However, if spatially dependent material parameters (gradient-index materials) are introduced, the field components are no longer independent from each other, and we are left with a coupled nonlinear eigenvalue problem.

Computational approaches for solving nonlinear eigenvalue problems have been studied in the literature [6, 7, 8, 9]. They often require specialized solvers not readily available in current finite element toolkits [10]. In this paper we pursue a different approach that allows to use well established, existing linear algebra techniques for solving linear eigenvalue problems. To that end, we investigate a general class of waveguide configurations that consist of spatially dependent material parameters and contain (arbitrarily shaped) interior conducting 2D material interfaces. In detail, our contributions are as follows:

\footnotetext{
${ }^{*}$ Corresponding author

Email addresses: songx762@umn.edu (Jung Heon Song), maier@math.tamu.edu (Matthias Maier), luskin@umn.edu (Mitchell Luskin)

${ }^{1}$ The authors were supported in part by ARO MURI Award W911NF-14-1-0247.

${ }^{2}$ The first and third author's research was supported in part by NSF Awards DMS1819220 and DMS-1906129.

${ }^{3}$ This author's research was supported in part by NSF Award DMS-1912847.
} 
- We derive a variational, nonlinear quartic eigenvalue problem for a waveguiding problem incorporating spatially dependent material parameters and interior conducting interfaces (see Section 2.2). The nonlinear quartic character of the eigenvalue problem stems from the fact that the spatially dependent material parameters cause a strong coupling between the otherwise decoupled transverse magnetic (TM) and transverse electric (TE) modes (as would normally be the case for the Helmholtz equation).

- We solve the quartic eigenvalue problem numerically by transforming it to a spectrally equivalent companion problem using a quadratification [11] approach. Additional numerical tools, such as the Möbius transform and a perfectly matched layer (PML) are employed to assist with solving the eigenvalue problem. We verify our numerical method against analytical solutions for prototypical geometries with internal conducting interfaces.

- As a practical example, we demonstrate how an improved quality factor (defined by the ratio of the real and the imaginary part of the computed eigenvalues) can be obtained (a) for a family of gradient-index host materials, and (b) by deformation of the geometry (see Section 5.3). Finally, we outline how our framework lays the groundwork for solving related shape optimization problems.

\subsection{Related works}

Optical properties of cylindrical waveguides with graphene interfaces have been extensively studied in the engineering community [3, 12, 13, 14]. Recently, focus has also shifted to gradient-index structures that couple with graphene [15, 16]. These structures are based on planar and cylindrical graphene-dielectric multilayer metamaterials, and have shown potential applications in terahertz imaging, sensing, detecting, and communication areas [15, 16]. In addition, the optimal design of graded-refractive index antireflection coatings has been investigated [17. The motivation behind our work is to formulate a numerical framework that is specifically designed for solving optical waveguiding problems with spatially dependent material parameters.

Numerical methods that compute eigenvalues of inhomogeneously loaded domains have been described before [18, 19, 20, 21]. For example, a finite difference frequency-domain method is used to analyze eigenmodes of inhomogeneously loaded rectangular waveguides in [18]. Another study [21] presents a method for computing solenoidal eigenmodes and the corresponding eigenvalues of the vector Helmholtz equation. We point out that a structurally 
similar nonlinear eigenvalue problem also arises in the context of quantum transmission problems described by the Schrödinger equation 22]: There, a fourth-order eigenvalue problem [22, Eq. (37)] emerges for a wave number that is then solved numerically by a linearized companion problem [22, Eq. (39)]. While similar in the resulting linear algebra structure to our waveguiding problem, neither of the above references directly address the question of eigenvalue problems with lower-dimensional conducting interfaces.

There exist a number of numerical methods for directly computing approximations of nonlinear eigenvalue problems. For example, the FEAST algorithm [23, 6] uses complex contour integration to compute a cluster of eigenvalues within some user-defined region in the complex plane. As such it is also well suited to compute solutions of quartic eigenvalue problems. It has been successfully used for simulating the propagation of light through optical fibers [7, 8]. Another numerical computing technique is based on the equivalent Rayleigh quotient optimization problem [9]. Here, a nonlinear eigenvalue problem is solved using a spectral transformation based on nonlinear shifting and a reformulation using second-order derivatives. In addition, an increasing number of mathematical software packages, such as for example SLEPc and the Julia programming language, provide black box solvers for polynomial eigenvalue problems $[24,25]$.

\subsection{Paper organization}

The remainder of the paper is organized as follows. In Section 2, we derive a variational quartic eigenvalue problem for the waveguiding problem based on time-harmonic Maxwell's equations. In Section 3, we describe our numerical approach for solving the quartic eigenvalue problem, including a linearization based on quadratification, the use of a Möbius transform to shift the spectrum, and a PML. Section 4 discusses and derives analytical solutions for prototypical configurations, which will be used to verify our numerical method in the subsequent section. Section 5 presents numerical results in domains with and without azimuthal symmetry. We demonstrate how our numerical method can be extended to arbitrary computational domains. Section 6 concludes the paper with a summary of our results and an outlook.

\section{Variational formulation}

We introduce a variational formulation for a relevant eigenvalue problem prescribed with a gradient-index host material with (arbitrarily shaped) con- 


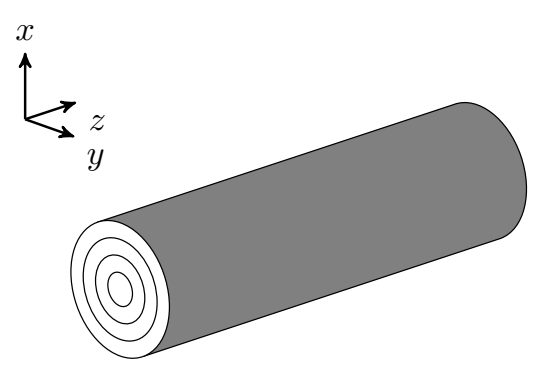

(a)

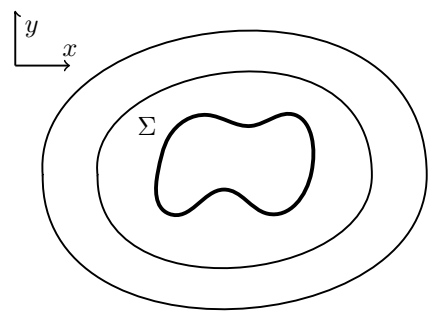

(b)

Figure 1: (a) Schematic of a prototypical multilayer waveguide. (b) Cross-sectional schematic of the computational domain. The closed curve, $\Sigma$, is prescribed with a nonvanishing conductivity. The waveguide is characterized by material parameters $\varepsilon(x, y)$ and $\mu(x, y)$.

ducting interfaces in the context of a waveguide configuration. A convenient rescaling of the equations to dimensionless forms is used [26].

\subsection{Preliminaries}

The source-free time-harmonic Maxwell's equations are given by

$$
\left\{\begin{aligned}
\nabla \times \boldsymbol{E} & =i \omega \mu \boldsymbol{H} \\
\nabla \times \boldsymbol{H} & =-i \omega \varepsilon \boldsymbol{E}
\end{aligned}\right.
$$

where $\boldsymbol{E}(\boldsymbol{x})$ and $\boldsymbol{H}(\boldsymbol{x})$ denote the electric and magnetic field, respectively, and $\omega$ is the temporal frequency. $\mu(x, y)$ and $\varepsilon(x, y)$ are complex-valued functions of the transverse coordinates, where $\mu$ denotes the magnetic permeability and $\varepsilon$ denotes the electric permittivity (see Figure 1). In order to study guided modes we make the additional ansatz

$$
\mathcal{F} \sim e^{i k_{z} z}
$$

and decompose the fields $\mathcal{F}=\{\boldsymbol{E}, \boldsymbol{H}\}$ and the gradient operator, $\nabla$, into their longitudinal and transverse parts, whence we obtain

$$
\mathcal{F}=\mathcal{F}_{s}+\hat{z} \mathcal{F}_{z}, \quad \nabla=\nabla_{s}+i k_{z} \hat{z}
$$

where the subscript $s$ denotes the transverse direction and $\hat{z}$ denotes the unit vector in $z$-direction. In the strong sense, (1) holds true everywhere except on the points comprising the conducting interface, $\Sigma$. The surface conductivity 
$\sigma^{\Sigma}(x, y)$ on the conducting interface $\Sigma$ gives rise to a jump condition on the tangential part of the magnetic field [26]. In summary, we obtain

$$
\left\{\begin{array}{l}
{\left[\boldsymbol{\nu} \times\left(\boldsymbol{H}_{s}+\hat{z} H_{z}\right)\right]_{\Sigma}=\sigma^{\Sigma}\left(\boldsymbol{\nu} \times\left(\boldsymbol{E}_{s}+\hat{z} E_{z}\right)\right) \times \boldsymbol{\nu},} \\
{\left[\boldsymbol{\nu} \cdot \mu\left(\boldsymbol{H}_{s}+\hat{z} H_{z}\right)\right]_{\Sigma}=0,} \\
{\left[\boldsymbol{\nu} \times\left(\boldsymbol{E}_{s}+\hat{z} E_{z}\right)\right]_{\Sigma}=0,} \\
{\left[\boldsymbol{\nu} \cdot \varepsilon\left(\boldsymbol{E}_{s}+\hat{z} E_{z}\right)\right]_{\Sigma}=\frac{1}{i \omega} \nabla \cdot\left(\sigma^{\Sigma} \boldsymbol{E}\right),}
\end{array}\right.
$$

where $\boldsymbol{\nu}$ is a chosen unit normal vector field on $\Sigma$, and $[.]_{\Sigma}$ denotes the jump over $\Sigma$ with respect to $\boldsymbol{\nu}$, viz.,

$$
[\boldsymbol{F}]_{\Sigma}(\boldsymbol{x}):=\lim _{\alpha \searrow 0}(\boldsymbol{F}(\boldsymbol{x}+\alpha \boldsymbol{\nu})-\boldsymbol{F}(\boldsymbol{x}-\alpha \boldsymbol{\nu})) \quad \boldsymbol{x} \in \Sigma .
$$

We also fix the notation

$$
\boldsymbol{F}^{+}:=\lim _{\alpha \searrow 0} \boldsymbol{F}(\boldsymbol{x}+\alpha \boldsymbol{\nu}), \quad \boldsymbol{F}^{-}:=\lim _{\alpha \searrow 0} \boldsymbol{F}(\boldsymbol{x}-\alpha \boldsymbol{\nu}), \quad \text { for } \boldsymbol{x} \in \Sigma .
$$

Next we introduce a convenient rescaling of the system to dimensionless form by setting the characteristic wavenumber of the ambient space to 1 [26]:

$$
\begin{gathered}
\boldsymbol{x} \rightarrow \breve{\boldsymbol{x}}=k_{0} \boldsymbol{x}, \quad \nabla_{s} \rightarrow \breve{\nabla}_{s}=\frac{1}{k_{0}} \nabla_{s}, \\
\mu \rightarrow \mu_{r}=\frac{1}{\mu_{0}} \mu, \quad \varepsilon \rightarrow \varepsilon_{r}=\frac{1}{\varepsilon_{0}} \varepsilon, \quad \sigma^{\Sigma} \rightarrow \sigma_{r}^{\Sigma}=\sqrt{\frac{\mu_{0}}{\varepsilon_{0}}} \sigma^{\Sigma} \\
\boldsymbol{E} \rightarrow \breve{\boldsymbol{E}}=\frac{k_{0}^{2}}{\omega \mu_{0}} \boldsymbol{E}, \quad \boldsymbol{H} \rightarrow \breve{\boldsymbol{H}}=k_{0} \boldsymbol{H}, \quad k_{z} \rightarrow \breve{k}_{z}=\frac{k_{z}}{k_{0}} .
\end{gathered}
$$

To lighten the notation, we omit the breve sign in the remainder of this paper. Applying the rescaling to 22 and rewriting into tangential and normal parts leads to the following interface conditions:

$$
\left\{\begin{aligned}
{\left[\boldsymbol{H}_{s}\right]_{\Sigma} \cdot \boldsymbol{\tau} } & =\left[\frac{i}{k_{s}^{2}}\left(k_{z} \partial_{\tau} H_{z}+\varepsilon_{r} \partial_{\nu} E_{z}\right)\right]_{\Sigma}=\sigma_{r}^{\Sigma} E_{z} \\
{\left[H_{z}\right]_{\Sigma} } & =-\sigma_{r}^{\Sigma} \boldsymbol{E}_{s} \cdot \boldsymbol{\tau}, \\
{\left[\mu_{r} \boldsymbol{H}_{s}\right]_{\Sigma} \cdot \boldsymbol{\nu} } & =\left[\frac{i \mu_{r}}{k_{s}^{2}}\left(k_{z} \partial_{\nu} H_{z}-\varepsilon_{r} \partial_{\tau} E_{z}\right)\right]_{\Sigma}=0 \\
{\left[\boldsymbol{E}_{s}\right]_{\Sigma} \cdot \boldsymbol{\tau} } & =\left[\frac{i}{k_{s}^{2}}\left(k_{z} \partial_{\tau} E_{z}-\mu_{r} \partial_{\nu} H_{z}\right)\right]_{\Sigma}=0 \\
{\left[E_{z}\right]_{\Sigma} } & =0 \\
{\left[\varepsilon_{r} \boldsymbol{E}_{s}\right]_{\Sigma} \cdot \boldsymbol{\nu} } & =\left[\frac{i \varepsilon_{r}}{k_{s}^{2}}\left(k_{z} \partial_{\nu} E_{z}+\mu_{r} \partial_{\tau} H_{z}\right)\right]_{\Sigma}=\frac{1}{i} \nabla_{s} \cdot\left(\sigma_{r}^{\Sigma} \boldsymbol{E}_{s}\right),
\end{aligned}\right.
$$


where $\partial_{\tau}$ and $\partial_{\nu}$ denote the derivative in the tangential and the normal direction, respectively; $k_{s}(x, y)^{2}=\mu_{r}(x, y) \varepsilon_{r}(x, y)-k_{z}^{2}$ is a function in the transverse direction; and where we have used the identities (see Appendix Appendix A

$$
\left\{\begin{aligned}
k_{s}^{2} \boldsymbol{E}_{s} & =i\left(k_{z} \nabla_{s} E_{z}+\mu_{r} \nabla_{s} \times H_{z}\right), \\
k_{s}^{2} \boldsymbol{H}_{s} & =i\left(k_{z} \nabla_{s} H_{z}-\varepsilon_{r} \nabla_{s} \times E_{z}\right) .
\end{aligned}\right.
$$

The first-order system (1) can be manipulated in a similar fashion (see Appendix A to obtain

$$
\left\{\begin{array}{l}
\nabla_{s} \times\left(\mu_{r}^{-1} \nabla_{s} \times \hat{z} E_{z}\right)+i k_{z} \nabla_{s} \times\left(\mu_{r}^{-1} \hat{z} \times \boldsymbol{E}_{s}\right)-\varepsilon_{r} E_{z}=0, \\
\nabla_{s} \times\left(\varepsilon_{r}^{-1} \nabla_{s} \times \hat{z} H_{z}\right)+i k_{z} \nabla_{s} \times\left(\varepsilon_{r}^{-1} \hat{z} \times \boldsymbol{H}_{s}\right)+\mu_{r} H_{z}=0 .
\end{array}\right.
$$

\subsection{Variational Statement}

Let $\Omega \subset \mathbb{R}^{n}$, where $n=2,3$, be a simply connected and bounded domain with Lipschitz-continous piecewise smooth boundary, $\partial \Omega$. Assume, in addition, that $\Sigma$ is a Lipschitz-continuous, piecewise smooth hypersurface. Let $\boldsymbol{\nu}$ and $\boldsymbol{\tau}$ denote the outer normal and the tangential vector on $\Sigma$ (see Figure 21. Some algebraic manipulation shows that $\nabla_{s} \times\left(\mu_{r}^{-1} \nabla_{s} \times \hat{z} E_{z}\right)=$ $-\nabla_{s} \cdot\left(\mu_{r}^{-1} \nabla_{s} \hat{z} E_{z}\right)$ and $\nabla_{s} \times\left(\mu_{r}^{-1} \hat{z} \times \boldsymbol{E}_{s}\right)=\hat{z} \nabla_{s} \cdot\left(\mu_{r}^{-1} \boldsymbol{E}_{s}\right)$, which can be used in conjunction with A.5) and (5) to obtain:

$$
\left\{\begin{array}{l}
-\nabla_{s} \cdot\left(\frac{\varepsilon_{r}}{k_{s}^{2}} \nabla_{s} E_{z}\right)-k_{z} \nabla_{s} \cdot\left(\frac{1}{k_{s}^{2}} \nabla_{s} \times \hat{z} H_{z}\right)-\varepsilon_{r} E_{z}=0, \\
-\nabla_{s} \cdot\left(\frac{\mu_{r}}{k_{s}^{2}} \nabla_{s} H_{z}\right)+k_{z} \nabla_{s} \cdot\left(\frac{1}{k_{s}^{2}} \nabla_{s} \times \hat{z} E_{z}\right)-\mu_{r} H_{z}=0 .
\end{array}\right.
$$

We observe that if the domain is homogeneously filled and isotropic, the curl terms vanish, yielding the familiar decoupled Helmholtz equation for $E_{z}$ and $H_{z}$. Now assume that

$$
\left[\varepsilon_{r}\right]_{\Sigma}=0, \quad\left[\mu_{r}\right]_{\Sigma}=0 .
$$

For the sake of brevity, we summarize the derivation here and refer the reader to Appendix A for details. We now want to remove the $k_{s}^{2}$ term in the denominator. Because $k_{s}(x, y)$ is a spatially dependent function, the lowest power of $k_{s}$ that achieves this goal is $k_{s}^{4}$. Thus, by multiplying (6) with $k_{s}^{4}$ and testing the first equation by $\varphi$ and the second equation by $\psi$, 
we obtain

$$
\begin{aligned}
& \left(\mu_{r} \varepsilon_{r}^{2} \nabla_{s} E_{z}, \nabla_{s} \varphi\right)+2\left(\varepsilon_{r} \nabla_{s} E_{z}, \nabla_{s}\left(\bar{\varepsilon}_{r} \bar{\mu}_{r}\right) \varphi\right) \\
& \quad+k_{z}\left(\mu_{r} \varepsilon_{r} \nabla_{s} \times \hat{z} H_{z}, \nabla_{s} \varphi\right)-k_{z}^{2}\left(\varepsilon_{r} \nabla_{s} E_{z}, \nabla_{s} \varphi\right) \\
& +2 k_{z}\left(\nabla_{s} \times \hat{z} H_{z}, \nabla_{s}\left(\bar{\varepsilon}_{r} \bar{\mu}_{r}\right) \varphi\right)-k_{z}^{3}\left(\nabla_{s} \times \hat{z} H_{z}, \nabla_{s} \varphi\right)-\left(\varepsilon_{r} k_{s}^{4} E_{z}, \varphi\right) \\
& \quad+\left(\varepsilon_{r} \mu_{r}^{2} \nabla_{s} H_{z}, \nabla_{s} \psi\right)+2\left(\mu_{r} \nabla_{s} H_{z}, \nabla_{s}\left(\bar{\varepsilon}_{r} \bar{\mu}_{r}\right) \psi\right) \\
& \quad-k_{z}\left(\mu_{r} \varepsilon_{r} \nabla_{s} \times \hat{z} E_{z}, \nabla_{s} \psi\right)-k_{z}^{2}\left(\mu_{r} \nabla_{s} H_{z}, \nabla_{s} \psi\right) \\
& -2 k_{z}\left(\nabla_{s} \times \hat{z} E_{z}, \nabla_{s}\left(\bar{\varepsilon}_{r} \bar{\mu}_{r}\right) \psi\right)+k_{z}^{3}\left(\nabla_{s} \times \hat{z} E_{z}, \nabla_{s} \psi\right)-\left(\mu_{r} k_{s}^{4} H_{z}, \psi\right) \\
& -i\left\langle\sigma_{r}^{\Sigma} k_{s}^{4} E_{z}, \varphi\right\rangle_{\Sigma}=0 .
\end{aligned}
$$

This shows the following statement.

Proposition 1. Provided that $k_{s} \neq 0$ and $\left[\varepsilon_{r}\right]_{\Sigma}=\left[\mu_{r}\right]_{\Sigma}=0$, the nonlinear eigenvalue problem

(N) Find $k_{z} \in \mathbb{C} \backslash\{0\}$ and $E_{z}, H_{z}$ s.t. (6) and (3) are satisfied can be restated as a quartic eigenvalue problem

(Q)

Find $k_{z} \in \mathbb{C} \backslash\{0\}$ and $E_{z}, H_{z} \in \boldsymbol{X}(\Omega)=\left\{(u, v): u, v \in H^{1}(\Omega, \mathbb{C})\right\}$ s.t.

$$
\mathcal{Q}\left(k_{z},\left(E_{z}, H_{z}\right)\right)[(\varphi, \psi)]=0 \quad \text { for all } \varphi, \psi \in H^{1}(\Omega, \mathbb{C}),
$$

where

$$
\mathcal{Q}\left(k_{z},\left(E_{z}, H_{z}\right)\right)[(\varphi, \psi)]=\sum_{l=0}^{4}\left(k_{z}\right)^{l} a_{l}\left(\left(E_{z}, H_{z}\right)\right)[(\varphi, \psi)]
$$


and

(9)

$$
\left\{\begin{array}{c}
a_{0}\left(\left(E_{z}, H_{z}\right),(\varphi, \psi)\right)=\left(\mu_{r} \varepsilon_{r}^{2} \nabla_{s} E_{z}, \nabla_{s} \varphi\right)+2\left(\varepsilon_{r} \nabla_{s} E_{z},\left(\nabla_{s} \bar{\mu}_{r} \bar{\varepsilon}_{r}\right) \varphi\right) \\
-\left(\mu_{r}^{2} \varepsilon_{r}^{3} E_{z}, \varphi\right)+\left(\varepsilon_{r} \mu_{r}^{2} \nabla_{s} H_{z}, \nabla_{s} \psi\right) \\
+2\left(\mu_{r} \nabla_{s} H_{z},\left(\nabla_{s} \bar{\mu}_{r} \bar{\varepsilon}_{r}\right) \psi\right) \\
-\left(\mu_{r}^{3} \varepsilon_{r}^{2} H_{z}, \psi\right)-i\left\langle\sigma_{r}^{\Sigma} \mu_{r}^{2} \varepsilon_{r}^{2} E_{z}, \varphi\right\rangle_{\Sigma} \\
a_{1}\left(\left(E_{z}, H_{z}\right),(\varphi, \psi)\right)=\left(\mu_{r} \varepsilon_{r} \nabla_{s} \times \hat{z} H_{z}, \nabla_{s} \varphi\right) \\
+2\left(\nabla_{s} \times \hat{z} H_{z},\left(\nabla_{s} \bar{\mu}_{r} \bar{\varepsilon}_{r}\right) \varphi\right) \\
-\left(\mu_{r} \varepsilon_{r} \nabla_{s} \times \hat{z} E_{z}, \nabla_{s} \psi\right) \\
-2\left(\nabla_{s} \times \hat{z} E_{z},\left(\nabla_{s} \bar{\mu}_{r} \bar{\varepsilon}_{r}\right) \psi\right) \\
a_{2}\left(\left(E_{z}, H_{z}\right),(\varphi, \psi)\right)=-\left(\varepsilon_{r} \nabla_{s} E_{z}, \nabla_{s} \varphi\right)+2\left(\mu_{r} \varepsilon_{r}^{2} E_{z}, \varphi\right) \\
-\left(\mu_{r} \nabla_{s} H_{z}, \nabla_{s} \psi\right)+2\left(\mu_{r}^{2} \varepsilon_{r} H_{z}, \psi\right) \\
+2 i\left\langle\sigma_{r}^{\Sigma} \mu_{r} \varepsilon_{r} E_{z}, \varphi\right\rangle_{\Sigma}, \\
a_{3}\left(\left(E_{z}, H_{z}\right),(\varphi, \psi)\right)=-\left(\nabla_{s} \times \hat{z} H_{z}, \nabla_{s} \varphi\right)+\left(\nabla_{s} \times \hat{z} E_{z}, \nabla_{s} \psi\right), \\
a_{4}\left(\left(E_{z}, H_{z}\right),(\varphi, \psi)\right)=-\left(\varepsilon_{r} E_{z}, \varphi\right)-\left(H_{z}, \psi\right)-i\left\langle\sigma_{r}^{\Sigma} E_{z}, \varphi\right\rangle_{\Sigma} .
\end{array}\right.
$$

\section{Numerical approach}

In this section, we outline our numerical approach for solving the quartic eigenvalue problem (Q). In particular we discuss a quadratification approach transforming the quartic eigenvalue problem into a companion problem with equivalent spectrum. A perfectly matched layer (PML), an artificial sponge layer placed near the boundary such that all outgoing waves decay exponentially, is introduced. The variational formulation (Q) is discretized on a non-uniform quadrilateral mesh.

Proposition 2. Let $\boldsymbol{X}_{h}(\Omega) \subset \boldsymbol{X}(\Omega)$ be a finite element subspace spanned by Lagrange finite elements $Q_{p}$.

$$
\sum_{l=0}^{4} k_{z}^{l} a_{l}\left(\left(E_{z}, H_{z}\right),(\varphi, \psi)\right)=0,
$$

Our goal is to translate $\mathrm{Q}_{h}$ into a finite dimensional linear problem, which then allows the use of a standard linear algebra solver. 


\subsection{Construction of a companion problem to the quartic eigenvalue problem}

We build upon the algebraic tool of quadratification introduced in [11], which allows us to reduce any even power matrix polynomial eigenvalue problem to a spectrally equivalent linear eigenvalue problem. Prop. 3 summarizes the main result (for a more general discussion of the ideas behind this reduction procedure, we refer the reader to [11, 27]).

Proposition 3 (Theorem 5.3 and 5.4 of [11, 27]). Consider a quartic eigenvalue problem, to find $\lambda \in \mathbb{C}$, and $x \in \mathbb{C}^{n}$, s.t.

$$
\sum_{k=0}^{4} \lambda^{k} A_{k} x=0
$$

where $A_{k} \in \mathbb{C}^{n \times n}$ are given matrices. Then, the linearization stated below is spectrally equivalent to the original problem (c.f. [11] Theorem 5.3 and 5.4): Find $\lambda \in \mathbb{C}$, and $z \in \mathbb{C}^{4 n}$ s.t.

$$
\left(\begin{array}{cccc}
A_{3} & 0 & -I_{n} & 0 \\
A_{1} & 0 & 0 & -I_{n} \\
0 & -I_{n} & 0 & 0 \\
A_{0} & 0 & 0 & 0
\end{array}\right) z+\lambda\left(\begin{array}{cccc}
A_{4} & 0 & 0 & 0 \\
A_{2} & I_{n} & 0 & 0 \\
0 & 0 & I_{n} & 0 \\
0 & 0 & 0 & I_{n}
\end{array}\right) z=0
$$

Here, $I_{n}$ denotes the $n \times n$ identity matrix.

With this result at hand, we rewrite $\left(\mathrm{Q}_{h}\right.$ as a linear eigenvalue problem:

$$
S z+\lambda M z=0
$$

where

$$
S=\left(\begin{array}{cccc}
a_{3} & 0 & -I_{n} & 0 \\
a_{1} & 0 & 0 & -I_{n} \\
0 & -I_{n} & 0 & 0 \\
a_{0} & 0 & 0 & 0
\end{array}\right), \quad M=\left(\begin{array}{cccc}
a_{4} & 0 & 0 & 0 \\
a_{2} & I & 0 & 0 \\
0 & 0 & I & 0 \\
0 & 0 & 0 & I
\end{array}\right), \quad z=\left(\begin{array}{c}
z_{1} \\
z_{2} \\
z_{3} \\
z_{4}
\end{array}\right)
$$

Here, by some abuse of notation $a_{i}$ denotes the corresponding matrix formed by the bilinear form $a_{i}(.$.$) given in (9)$ and by fixing a basis of $\boldsymbol{X}_{h}(\Omega)$. A quick computation shows that the eigenvectors of the original problem $\left(\mathrm{Q}_{h}\right)$ and of the final linearized problem $\left(\mathrm{LQ}_{h}\right)$ are related as follows. 


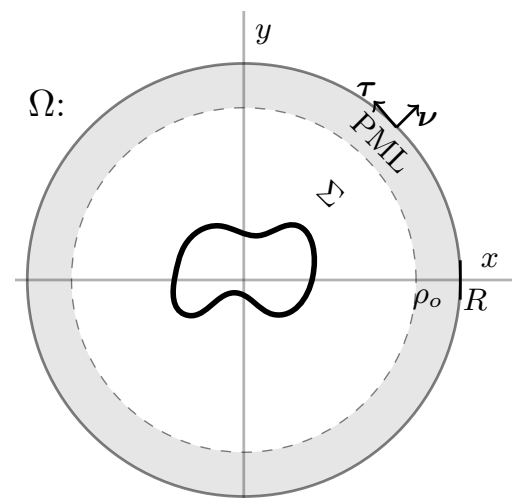

Figure 2: Schematic of the computational domain of a circular waveguide $\Omega$, with boundary $\partial \Omega$, outer normal vector $\boldsymbol{\nu}$, and tangential vector $\boldsymbol{\tau}$. A conducting ciruclar interface is labeled $\Sigma$. A perfectly matched layer (PML) is enforced in the shaded region.

Proposition 4. Let $\lambda \in \mathbb{C}$ and $x \in \mathbb{C}^{n}$ be an eigenvalue with corresponding eigenvector of $\left(\mathrm{Q}_{h}\right)$. Then, $\lambda$ and $z \in \mathbb{C}^{4 n}$ given by

$$
\left\{\begin{array}{l}
z_{1}=\lambda x \\
z_{2}=\lambda^{2}\left(a_{3}+\lambda a_{4}\right) x \\
z_{3}=\lambda\left(a_{3}+\lambda a_{4}\right) x \\
z_{4}=-a_{0} x
\end{array}\right.
$$

is an eigenvalue with corresponding eigenvector of (LQh $)$. Conversely, if $\lambda \in \mathbb{C}$ and $z \in \mathbb{C}^{4 n}$ is an eigenvalue and eigenvector pair of $\left(\mathrm{LQ}_{h}\right)$, then provided that $\operatorname{det}\left(a_{0}\right) \neq 0$ and $\operatorname{det}\left(\lambda a_{4}+a_{3}\right) \neq 0$, the vector $z$ is characterized by (11) and $\lambda$ and $x$ are an eigenvalue and eigenvector pair of $\left(\mathrm{Q}_{h}\right)$.

\subsection{Perfectly Matched Layer}

A perfectly matched layer (PML) is a truncation procedure motivated from electromagnetic scattering problems in the time domain. The idea is to surround the computational domain with a so-called sponge layer, an artificial boundary wherein all outgoing electromagnetic waves decay exponentially with minimal artificial reflection (see Figure 22). As outlined in $[28,29,26$, we carry out a change of coordinates from the computational domain with real-valued coordinates to a domain with complex-valued coordinates. We refer the reader to [28] for details. For a spherical absorption layer, we define the transformation $\tilde{\rho}=\rho \bar{d}$, where

$$
d=1+i s(r), \quad \bar{d}=1+i / r \int_{\rho}^{r} s(\tau) d \tau
$$


and $s(\tau)$ is an appropriately chosen, nonnegative scaling function. Applying the above transformation, the quartic eigenvalue problem takes the following rescaled form within the PML:

$$
\begin{array}{r}
\hat{\nabla}_{s} \cdot\left(k_{s}^{2}\left(\varepsilon_{r} \hat{\nabla}_{s} \hat{E}_{z}\right)\right)-2 k_{s}^{2} \hat{\nabla}_{s} \cdot\left(\varepsilon_{r} \hat{\nabla}_{s} \hat{E}_{z}\right)+k_{z} \hat{\nabla}_{s} \cdot\left(k_{s}^{2} \hat{\nabla}_{s} \times \hat{H}_{z}\right) \\
-2 k_{z} k_{s}^{2} \hat{\nabla}_{s} \cdot\left(\hat{\nabla}_{s} \times \hat{H}_{z}\right)+\hat{\nabla}_{s} \cdot\left(k_{s}^{2}\left(\mu_{r} \hat{\nabla}_{s} \hat{H}_{z}\right)\right)-2 k_{s}^{2} \hat{\nabla}_{s} \cdot\left(\mu_{r} \hat{\nabla}_{s} \hat{H}_{z}\right) \\
+k_{z} \hat{\nabla}_{s} \cdot\left(k_{s}^{2} \hat{\nabla}_{s} \times \hat{E}_{z}\right)+2 k_{z} k_{s}^{2} \hat{\nabla}_{s} \cdot\left(\hat{\nabla}_{s} \times \hat{E}_{z}\right) \\
-\varepsilon_{r} k_{s}^{4} \hat{E}_{z}-\mu_{r} k_{s}^{4} \hat{H}_{z}=0 .
\end{array}
$$

This can be rewritten as

$$
\begin{array}{r}
\frac{1}{d \bar{d}} \nabla_{s} \cdot\left(k_{s}^{2} \varepsilon_{r} A \nabla_{s} E_{z}\right)-\frac{2 k_{s}^{2}}{d \bar{d}} \nabla_{s} \cdot\left(\varepsilon_{r} A \nabla_{s} E_{z}\right)+\frac{k_{z}}{d \bar{d}} \nabla_{s} \cdot\left(k_{s}^{2} \nabla_{s} \times H_{z}\right) \\
-\frac{2 k_{z} k_{s}^{2}}{d \bar{d}} \nabla_{s} \cdot\left(\nabla_{s} \times H_{z}\right)+\frac{1}{d \bar{d}} \nabla_{s} \cdot\left(k_{s}^{2} \varepsilon_{r} A \nabla_{s} E_{z}\right)-\frac{2 k_{s}^{2}}{d \bar{d}} \nabla_{s} \cdot\left(\varepsilon_{r} A \nabla_{s} E_{z}\right) \\
+\frac{k_{z}}{d \bar{d}} \nabla_{s} \cdot\left(k_{s}^{2} \nabla_{s} \times H_{z}\right)+\frac{2 k_{z} k_{s}^{2}}{d \bar{d}} \nabla_{s} \cdot\left(\nabla_{s} \times E_{z}\right) \\
+\varepsilon_{r} k_{s}^{4} E_{z}+\mu_{r} k_{s}^{4} H_{z}=0
\end{array}
$$

where $A=T_{\boldsymbol{e}_{x}, \boldsymbol{e}_{r}}^{-1} \operatorname{diag}(\bar{d} / d, d / \bar{d}) T_{\boldsymbol{e}_{x}, \boldsymbol{e}_{r}}$, and $T_{\boldsymbol{e}_{x}, \boldsymbol{e}_{r}}$ is the rotation matrix that rotates $\boldsymbol{e}_{r}$ onto $\boldsymbol{e}_{x}$. We enforce the condition that the material parameters are constant outside the PML, i.e., $\varepsilon_{r}$ and $\mu_{r}$ do not undergo a change of coordinate. Additionally, because the eigenmodes of our interest are confined to the conducting interface, which is situated inside the PML, no coordinate change is needed for the jump condition. The modified bilinear forms, $\tilde{a}_{i}$, 
are

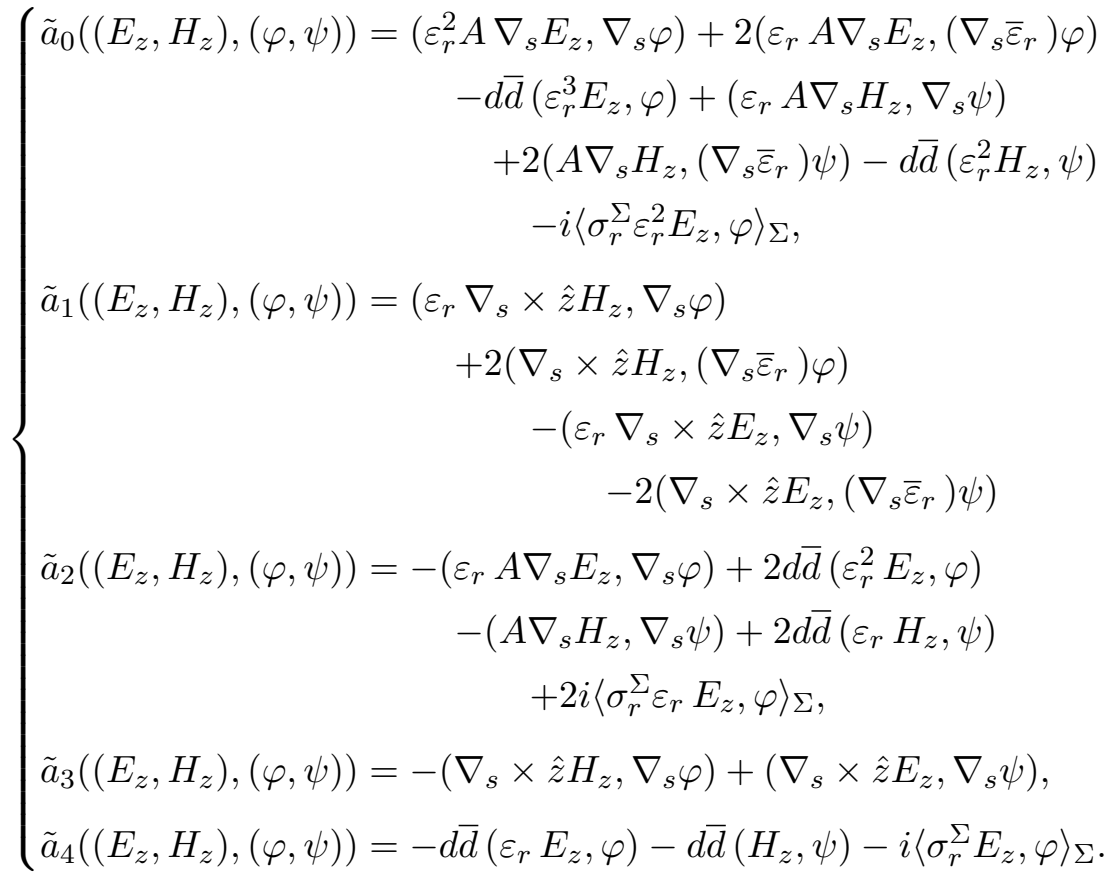

\subsection{Möbius Transform}

Numerical computations of Maxwell eigenvalue problems, in particular with a perfectly matched layer, often contain a large number of spurious eigenvalues. Spurious modes are numerical solutions of the vector wave equation that convey no physical meaning. A number of different approaches have been proposed to eliminate part or all of the spurious modes, e.g., by enforcing the solenoidal nature of the flux [30], by adding a penalty factor [31, 32], or by solving Maxwell's equations via the method of constraints [33, 34, 35. A readily implementable approach that best suits our computational setup is through the Mobius transformation, which shifts the spectrum in such a way that the modes of interest are close to the origin. They can then be selectively computed with conventional Krylov-space iteration techniques. The Möbius transform is a conformal mapping, defined as follows.

$$
\lambda \mapsto \frac{a \lambda+b}{c \lambda+d},
$$

where $a, b, c, d \in \mathbb{C}$ are chosen parameters. Over arbitrary fields, the Möbius transformation preserves a number of spectral features of matrix polynomials, such as regularity, rank, minimal indicies, the location of zero entries, 
symmetry, and skew-symmetry [36]. In particular, every Möbius transformation preserves the relation of spectral equivalence [36]. The computational eigenvalue problem, after introducing a PML, truncating the domain, and applying a finite element disretization, can be written as

$$
S z=k_{z} M z,
$$

for a complex-valued vector $z$ and appropriate complex-valued matrices $S$ and $M$. The implementation of the PML discussed in 3.2 necessitates changes to the definition of $a_{1}, a_{2}, a_{3}$, and $a_{4}$. The idea is to use the Möbius transform to map points near the origin to target values

$$
(a S+b M) z=\tilde{k}_{z}(c S+d M) z,
$$

where $a, b, c, d$ are the Möbius transform parameters. The original eigenvalue can be retrieved via the inverse Möbius transform $k_{z}=\left(-b+d \tilde{k}_{z}\right) /\left(a-c \tilde{k}_{z}\right)$.

\section{Validation of weak formulation}

In this section, the analytical solution for constant material parameters is derived and discussed. We use the analytic result to validate our numerical approach.

By assuming constant material parameters, the quartic eigenvalue problem Q does not exhibit any hybridization and reduces to a linear eigenvalue problem: Find $u \in H^{1}(\Omega, \mathbb{C})$ s.t.

$$
\mathcal{L}(u)[\varphi]:=a(u, \varphi)+k_{z}^{2} m(u, \varphi),
$$

for $\varphi \in H^{1}(\Omega, \mathbb{C})$ and where we have introduced the bilinear forms

$$
\left\{\begin{array}{l}
a(u, \varphi)=\int_{\Omega} \nabla_{s} u \cdot \nabla_{s} \bar{\varphi} \mathrm{d} x-\int_{\Omega} \mu_{r} \varepsilon_{r} u \bar{\varphi} \mathrm{d} x-i \int_{\Sigma} \mu_{r} \sigma_{r}^{\Sigma} u \bar{\varphi} \mathrm{d} o_{x}, \\
m(u, \varphi)=\int_{\Omega} u \bar{\varphi} \mathrm{d} x+i \int_{\Sigma} \sigma_{r}^{\Sigma} \varepsilon_{r}^{-1} u \bar{\varphi} \mathrm{d} o_{x} .
\end{array}\right.
$$

For a simple spherical geometry that is rotationally invariant, the field solution can be expressed as a superposition of the modified Bessel functions of the first and second kind. In the case of a waveguide with a single circular interface $\Sigma$, i.e., where $\Sigma$ is described by a circle with origin 0 and radius $\rho_{i}$, the analytical solution takes the following form.

$$
\begin{aligned}
& E_{z}= \begin{cases}A_{m} I_{m}\left(i k_{s} \rho\right) e^{i m \theta} e^{i k_{z} z} & \text { for } \rho<\rho_{i}, \\
B_{m} K_{m}\left(i k_{s} \rho\right) e^{i m \theta} e^{i k_{z} z} & \text { for } \rho>\rho_{i},\end{cases} \\
& H_{z}= \begin{cases}C_{m} I_{m}\left(i k_{s} \rho\right) e^{i m \theta} e^{i k_{z} z} & \text { for } \rho<\rho_{i}, \\
D_{m} K_{m}\left(i k_{s} \rho\right) e^{i m \theta} e^{i k_{z} z} & \text { for } \rho>\rho_{i},\end{cases}
\end{aligned}
$$


where $I_{m}$ and $K_{m}$ denote the modified Bessel functions of the first and second kind, respectively, and $A_{m}, B_{m}, C_{m}$, and $D_{m}$ are constants that are determined by the boundary conditions [13, 3]. Assuming that the conducting film is located on the boundary of the interior circle with radius $\rho_{i}$, we equate the jump conditions (3) of each field component. Then (3) reduces to the following algebraic condition, from which we can retrieve the propagation constant, $k_{z}$ :

$$
\operatorname{det}\left(M-\sigma_{r}^{\Sigma} N\right)=0
$$

where

$$
M:=\left(\begin{array}{cccc}
I_{m}\left(i k_{s} R\right) & 0 & -K_{m}\left(i k_{s} R\right) & 0 \\
\frac{k_{z} m I_{m}\left(i k_{s} R\right)}{R k_{s}^{2}} & -\frac{\mu I_{m}^{\prime}\left(i k_{s} R\right)}{k_{s}} & -\frac{k_{z} m K_{m}\left(i k_{s} R\right)}{R k_{s}^{2}} & \frac{\mu K_{m}^{\prime}\left(i k_{s} R\right)}{k_{s}} \\
\frac{\epsilon_{1} I_{m}^{\prime}\left(i k_{s} R\right)}{k_{s}} & \frac{k_{z} m I_{m}\left(i k_{s} R\right)}{R k_{s}^{2}} & -\frac{\epsilon_{2} K_{m}^{\prime}\left(i k_{s} R\right)}{k_{s}} & -\frac{k_{z} m K_{m}\left(i k_{s} R\right)}{R k_{s}^{2}} \\
0 & I_{m}\left(i k_{s} R\right) & 0 & -K_{m}\left(i k_{s} R\right)
\end{array}\right)
$$

and

$$
N:=\left(\begin{array}{cccc}
0 & 0 & 0 & 0 \\
0 & 0 & 0 & 0 \\
I_{m}\left(i k_{s} R\right) & 0 & 0 & 0 \\
-\frac{k_{z} m I_{m}\left(i k_{s} R\right)}{R k_{s}^{2}} & \frac{\mu I_{m}^{\prime}\left(i k_{s} R\right)}{k_{s}} & 0 & 0
\end{array}\right)
$$

We solve for the zeros of (18) numerically via a root finding algorithm for modal orders $m=1,2$ and 3 . The computed values are then compared against those of the linear problem $(\mathrm{L})$ and of the quartic problem (Q) (see Table 1 and Figure 3). We now validate our numerics with the analytical results. Three validations are carried out: analytical, numerical with linear eigenvalue, and lastly, the quartic eigenvalue problem. For simplicity, we assume the computational domain is isotropic, with material parameters $\varepsilon_{r}=\mu_{r} \equiv 1$. A conducting interface is coated on the boundary of the interior circle with radius $\rho_{i}=0.3$, on a circular domain with radius $R=2.0$. The surface conductivity is set to $\sigma_{r}^{\Sigma}=0.002+0.20 i$.

The analytic computation of the propagation constant $k_{z}$ requires finding the complex roots of the determinant of the $4 \times 4$ matrix. We will consider modal orders of $m=1,2$ and 3 to ease the computation. The computational 


\begin{tabular}{|c|c|c|c|}
\hline & Eigenvalues from $\mathrm{L}$ & Eigenvalues from $Q$ & Eigenvalues from 18 \\
\hline Mode & $k_{z}$ & $k_{z}$ & $k_{z}$ \\
\hline 1 & $9.447 \pm 0.090468 i$ & $9.447 \pm 0.090467 i$ & $9.447 \pm 0.090467 i$ \\
\hline 2 & $13.00 \pm 0.090641 i$ & $13.00 \pm 0.090640 i$ & $13.00 \pm 0.090641 i$ \\
\hline 3 & $16.17 \pm 0.099812 i$ & $16.17 \pm 0.099812 i$ & $16.17 \pm 0.099813 i$ \\
\hline
\end{tabular}

Table 1: Validation of computed eigenvalues form the linear problem 15, the quartic problem (Q), and the analytical approach $\sqrt{18}$ for a single-layer waveguide. Note that the values obtained are in agreement with confidence level of less than $1 \%$. Parameters used are $R=2.0, \sigma_{r}^{\Sigma}=0.002+0.20 i, \varepsilon_{r}=\mu_{r} \equiv 1$, and $\rho_{i}=0.3$.

results displayed in Table 1 deviate by less than $1 \%$ from each other. We can thus expect a confidence level of $1 \%$ or better in our numerical computations. Figure 3 shows the intensity of the numerically computed electric field, $\left\|E_{z}\right\|_{2}$, and a comparison of, both, the analytic and numerical solutions. We thus conclude that our numerical framework is a reliable model that can effectively simulate hybrid plasmonic modes.

\section{Eigenvalue computations of the linearized companion problem}

In this section, we present a number of computational results obtained from solving the quartic eigenvalue problem (Q) for a class of prototypical waveguides with gradient-index materials. We examine numerically how the spectrum of such a hybridized configuration behaves under modification of spatially dependent material parameters, $\varepsilon_{r}(\rho, \theta)$. We further investigate the relationship between mesh deformation and the quality factor (defined as the ratio of real part of the eigenvalue to imaginary part), and study the degree by which the spectrum changes. All numerical computations are carried out with the finite element library deal.II [10]. We use a Krylov-Schur method to compute solutions of the linearized eigenproblem $\left(\mathrm{LQ}_{h}\right][24]$.

We demonstrate numerically how it is possible to attain an improved quality factor by prescribing the host material with a radially-varying refractive index profile. This is methodically carried out in the subsequent subsections. First, a parameter study is conducted to validate our choice of discretization parameters. Second, we solve the quartic eigenvalue problem (LQh using a number of permittivity functions, and observe how the spectrums differ from those obtained in an isotropic medium. Lastly, we deform our computational domain to demonstrate that our numerical framework is equipped to handle even the most general configuration. The key idea behind this generalization is to show that we can manipulate the spectrum 


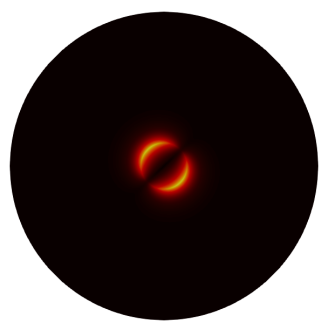

(a) $m=1$

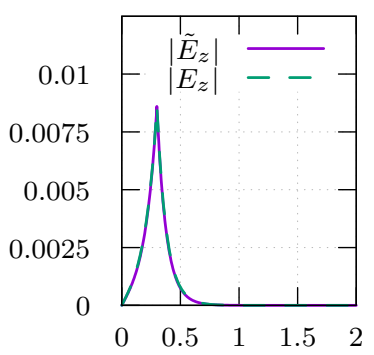

(d) $m=1$

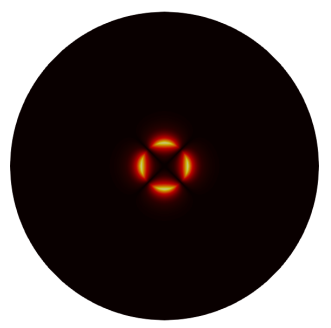

(b) $m=2$

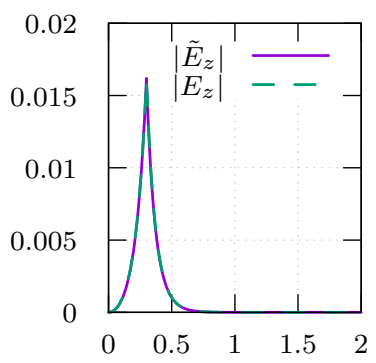

(e) $m=2$

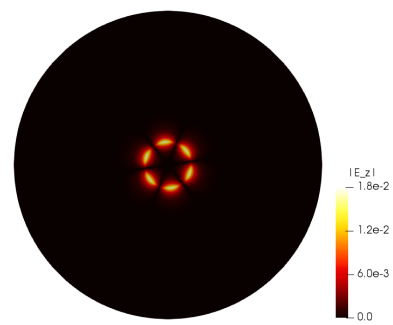

(c) $m=3$

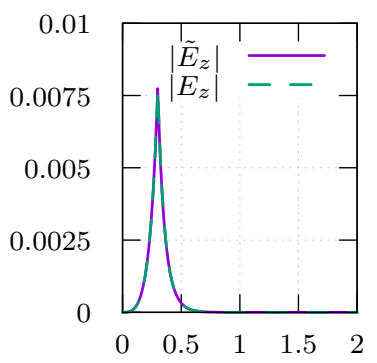

(f) $m=3$

Figure 3: Computational results for modal order $m=1,2,3$ for a spherical reference configuration with constant material parameters, and prescribed conductivity $\sigma_{r}^{\Sigma}=0.002+0.20 i$ at radius $\rho_{i}=0.3$. The computational domain is a disc with radius $R=2.0$. (a-c) shows the magnitude of the numerically computed electric field, $\tilde{E}_{z}$. The computed electric field is compared against an analytic solution, $E_{z}$, in (d)-(f).

by manipulating the shape. We make note of the evolution of eigenmodes, and how our framework can be used as a basis for shape optimization of gradient-index waveguides.

The spectrum is computed numerically by using SLEPc [24], a general purpose eigensolver built on top of PETSc [37]. The eigensolver provides a number of Krylov-space methods, such as the Arnoldi, Lanczos, KrylovSchur, and conjugate-gradient methods. For our purposes, we make use of the Krylov-Schur method for its faster convergence.

\subsection{Validation of discretization parameters}

The computational domain, $\Omega$, is chosen to be the circle with radius 1. A spherical PML is enforced for $\rho>0.8$. The surface conductivity $\sigma_{r}^{\Sigma}=0.002+0.20 i$ is chosen that is within a realistic parameter range [26] and is located at $\rho_{i}=0.3$. Following [29], the nonnegative scaling function 


\begin{tabular}{|c|c|c|c|c|c|c|}
\hline \multirow[b]{2}{*}{ Mode } & \multicolumn{3}{|c|}{$s_{o}=1.5, R=1.0, n=4, \mathrm{DoF}=19138$} & \multicolumn{3}{|c|}{$s_{o}=1.5, R=1.0, n=6, \mathrm{DoF}=303874$} \\
\hline & $\operatorname{Re} k_{z}$ & $\operatorname{Im} k_{z}$ & $\operatorname{Re} k_{z} / \operatorname{Im} k_{z}$ & $\operatorname{Re} k_{z}$ & $\operatorname{Im} k_{z}$ & $\operatorname{Re} k_{z} / \operatorname{Im} k_{z}$ \\
\hline 1 & 30.5400 & 0.17646 & 173.070 & 30.4160 & 0.17540 & 173.409 \\
\hline 2 & 40.5520 & 0.21898 & 185.186 & 40.5043 & 0.21881 & 185.112 \\
\hline
\end{tabular}

(a) Vary the number of refinements, $n$.

\begin{tabular}{rrrc}
\hline & \multicolumn{3}{c}{$s_{o}=1.5, R=1.0, n=5, \mathrm{DoF}=76162$} \\
\cline { 2 - 4 } Mode & $\operatorname{Re} k_{z}$ & $\operatorname{Im} k_{z}$ & $\operatorname{Re} k_{z} / \operatorname{Im} k_{z}$ \\
1 & 30.4160 & 0.17582 & 172.995 \\
2 & 40.5043 & 0.21881 & 185.112 \\
\hline
\end{tabular}

(b) The control case for the parameter studies.

\begin{tabular}{|c|c|c|c|c|c|c|}
\hline \multirow[b]{2}{*}{ Mode } & \multicolumn{3}{|c|}{$s_{o}=1.5, R=0.5, n=5, \mathrm{DoF}=76162$} & \multicolumn{3}{|c|}{$s_{o}=1.5, R=2.0, n=5, \mathrm{DoF}=76162$} \\
\hline & $\operatorname{Re} k_{z}$ & $\operatorname{Im} k_{z}$ & $\operatorname{Re} k_{z} / \operatorname{Im} k_{z}$ & $\operatorname{Re}_{z}$ & $\operatorname{Im} k_{z}$ & $\operatorname{Re} k_{z} / \operatorname{Im} k_{z}$ \\
\hline 1 & 30.4242 & 0.17528 & 173.575 & 30.8567 & 0.17331 & 178.043 \\
\hline 2 & 40.4905 & 0.21876 & 185.091 & 40.5043 & 0.21881 & 185.112 \\
\hline
\end{tabular}

(c) Vary the domain size, $R$.

Table 2: Validation of discretization parameters: Parameter study with permittivity function $\varepsilon_{r}(\boldsymbol{x})=3 \cdot \chi_{|\boldsymbol{x}|<\rho_{i}}+1 \cdot \chi_{\rho_{i}<|\boldsymbol{x}|<R} \cdot \mu_{r}(\boldsymbol{x}) \equiv 1$ and $\sigma_{r}^{\Sigma}=0.002+0.20 i$. (b) is the control discretization parameters. (a) differs from (b) in the number of refinements, and (c) differs from (b) in the size of the domain.

$s(\rho)$ is chosen to be

$$
s(\rho)=s_{0} \frac{(\rho-0.8 R)^{2}}{(R-0.8 R)^{2}}
$$

where we set the free parameter $s_{0}$ to be $s_{0}=1.5$ in our computations. We carry out a parameter study to test the validity and the sensitivity of discretization parameters. Table 2 summarizes the parameter study quantitatively. As can be seen, the eigenmodes comptued are stable with respect to variations of PML strength $s_{0}$, the number of initial refinements $n$, and domain sizes $R$. A spectral transformation is carried out in the form of the Möbius transformation, with the parameters chosen to be $a=1, b=$ $-10, c=1, d=10$. We conclude that $R=1.0$ and $k=5$ is a valid choice of discretization parameters. 


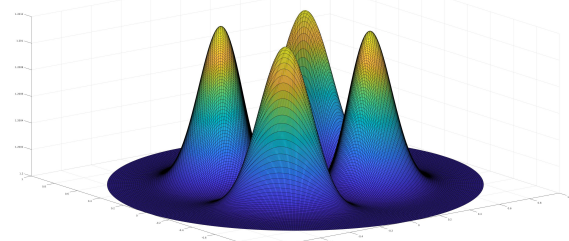

(a) Surface plot of $\epsilon_{r, 1}$

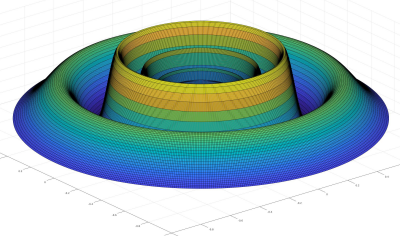

(b) Surface plot of $\epsilon_{r, 2}$

Figure 4: A surface plot of the two permittivity profiles used in the computations. (a) a $3 \mathrm{D}$ plot of $\varepsilon_{r, 1}(x, y)$ as given by [20; (b) a 3D plot of $\varepsilon_{r, 2}(x, y)$ as given by 20].

\subsection{Gradient-index waveguide}

Our numerical framework admits any (locally) differentiable material parameters. To demonstrate this, we consider the following model material permittivity functions and analyze their spectrums in relation to those of the isotropic medium.

$$
\left\{\begin{array}{c}
\varepsilon_{r, 1}(\rho, \theta)=1.2+\frac{1}{2} \sin \left(\frac{\rho^{2}}{\left(2 \rho_{i}\right)^{2}} \sin 2 \theta\right) \exp \left(-\frac{\left(\rho-2 \rho_{i}\right)^{2}}{\left(2 \rho_{i}\right)^{2}}\right), \\
\varepsilon_{r, 2}(\rho)=1+\left(\frac{\rho}{2}+\rho^{2} \sin \left(\frac{2 \pi}{\rho}\right)\right) \exp \left(-\frac{\left(\rho-2 \rho_{i}\right)^{2}}{2 \rho_{i}}\right),
\end{array}\right.
$$

where $\rho_{i}$ is the radius at which the conducting interface is situated for a circular waveguide. We set $\rho_{i}=0.1$ for our computations. The surface plot of these profiles can be seen in Figure 4 . The key aspect of these functions is that $\varepsilon_{r}$ remains constant in the PML, which enables us to implement the PML as laid out in 3.2 . The computations are carried out using the unit circular waveguide. For comparison, we plot the eigenvalues for both isotropic media and materials defined by (20) (see Figure 5). The quality factor, $\eta=\operatorname{Re} k_{z} / \operatorname{Im} k_{z}$, of the first 5 modes of each of these functions are laid out in Table 3 .

We note of a few observations. The eigenvalues obtained from $\varepsilon_{r, i}$ are more clustered than those from isotropic media. Even though the range of $\varepsilon_{r, i}$ $\left(\varepsilon_{r, 1} \in(1.0,1.4)\right.$ and $\left.\varepsilon_{r, 2} \in(1.0,1.1614)\right)$ is relatively close to 1 , we observe significant changes to the spectrum and the quality factor. From Table 3 , a much longer propagation is observed for $\varepsilon_{r, 2}$ than for $\varepsilon_{r, 1}$, despite their relatively similar range. This demonstrates that the relationship between $\varepsilon_{r}$ and the quality factor, $\eta$, is not trivial, and suggests that it is indeed possible to improve $\eta$ with a nontrivial gradient-index $\varepsilon_{r}$. 


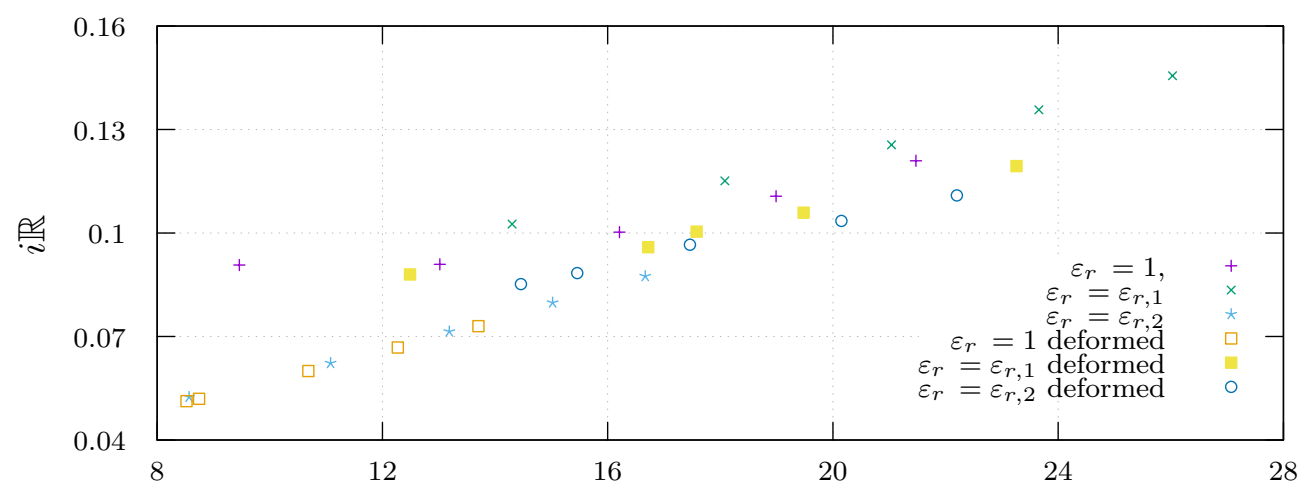

Figure 5: Spectrums computed using different $\varepsilon \mathbb{R}$ for spherical and deformed domain. For $\varepsilon_{r} \equiv 1$ and $\varepsilon_{r, 1}$, both the real and the imaginary parts have decreased after the domain was deformed. The opposite is observed for $\varepsilon_{r, 2}$. In the spherical domain case, the eigenvalues for $\varepsilon_{r} \equiv 1$ and $\varepsilon_{r, 1}$ remain relatively close to one another, but undergo a noticeable difference after the mesh deformation. The opposite can be seen for $\varepsilon_{r, 1}$ and $\varepsilon_{r, 2}$.

\subsection{Generalized configuration}

As a final numerical investigation, we compute a deformed geometric configuration by using the same permittivity functions introduced in the preceding section. The purpose of this exercise is two-fold. For one, we demonstrate that our computational approach can handle large mesh deformations. Secondly, we demonstrate with this computation that the quality factor $\eta$ can indeed be controlled and optimized by changing the shape of the waveguide. This leads to a number of very appealing shape optimization problems.

Let us introduce a mapping $\delta=\left(\delta_{x}, \delta_{y}\right)$ that deforms the mesh near

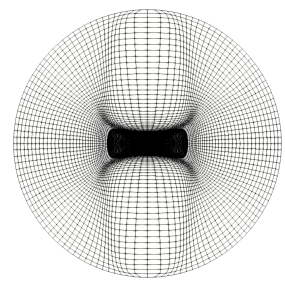

(a) Deformed mesh

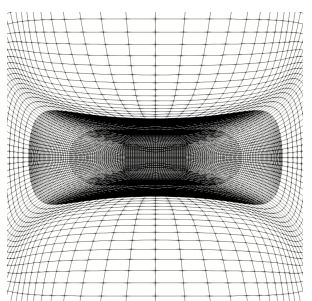

(b) zoom

Figure 6: The deformed mesh (a) obtained with the mapping function $\delta$ outlined below. The mesh has a total number of around 370,000 cells. Additional refinements are a priori enforced around the conducting interface. 


\begin{tabular}{|c|c|c|c|c|c|c|c|c|c|}
\hline \multirow[b]{2}{*}{ Mode } & \multicolumn{3}{|c|}{$\varepsilon_{r} \equiv 1$} & \multicolumn{3}{|c|}{$\varepsilon_{r}=\varepsilon_{r, 1}$} & \multicolumn{3}{|c|}{$\varepsilon_{r}=\varepsilon_{r, 2}$} \\
\hline & $\operatorname{Re} k_{z}$ & $\operatorname{Im} k_{z}$ & $\eta_{3}$ & $\operatorname{Re} k_{z}$ & $\operatorname{Im} k_{z}$ & $\eta$ & $\operatorname{Re} k_{z}$ & $\operatorname{Im} k_{z}$ & $\eta$ \\
\hline 1 & 13.02 & 0.09093 & 143.2 & 14.30 & 0.1026 & 139.4 & 8.569 & 0.05243 & 163.4 \\
\hline 2 & 16.21 & 0.1003 & 161.7 & 18.08 & 0.1151 & 157.1 & 11.08 & 0.06230 & 177.9 \\
\hline 3 & 18.99 & 0.1107 & 171.6 & 21.04 & 0.1256 & 167.6 & 13.19 & 0.07144 & 184.6 \\
\hline 4 & 21.47 & 0.1210 & 177.6 & 23.66 & 0.1357 & 174.3 & 15.02 & 0.07979 & 188.3 \\
\hline 5 & 23.74 & 0.1309 & 181.4 & 26.03 & 0.1456 & 178.8 & 16.67 & 0.08746 & 190.6 \\
\hline
\end{tabular}

(a) Eigenvalues for spherical domain

\begin{tabular}{|c|c|c|c|c|c|c|c|c|c|}
\hline \multirow[b]{2}{*}{ Mode } & \multicolumn{3}{|c|}{$\varepsilon_{r} \equiv 1$} & \multicolumn{3}{|c|}{$\varepsilon_{r}=\varepsilon_{r, 1}$} & \multicolumn{3}{|c|}{$\varepsilon_{r}=\varepsilon_{r, 2}$} \\
\hline & $\operatorname{Re} k_{z}$ & $\operatorname{Im} k_{z}$ & $\eta_{3}$ & $\operatorname{Re} k_{z}$ & $\operatorname{Im} k_{z}$ & $\eta$ & $\operatorname{Re} k_{z}$ & $\operatorname{Im} k_{z}$ & $\eta$ \\
\hline 1 & 8.521 & 0.05126 & 166.2 & 12.49 & 0.0880 & 141.9 & 14.46 & 0.0852 & 169.8 \\
\hline 2 & 8.745 & 0.05194 & 168.8 & 16.72 & 0.0959 & 163.8 & 15.46 & 0.0884 & 174.9 \\
\hline 3 & 10.69 & 0.06003 & 178.0 & 17.58 & 0.1004 & 175.1 & 17.46 & 0.0966 & 182.1 \\
\hline 4 & 12.27 & 0.06683 & 183.7 & 19.48 & 0.1059 & 184.0 & 20.15 & 0.1035 & 194.8 \\
\hline 5 & 13.71 & 0.07300 & 187.8 & 23.26 & 0.1194 & 194.8 & 22.20 & 0.1109 & 200.2 \\
\hline
\end{tabular}

(b) Eigenvalues for deformed domain

Table 3: Eigenvalues $k_{z}$ and quality factor $\eta=\operatorname{Re} k_{z} / \operatorname{Im} k_{z}$ for different $\varepsilon_{r}$ in spherical and deformed domains. Noticeable shift in the spectrums is observed from (a) to (b), which in turn, has led to non-negligible increases in $\eta$.

the conducting interface heavily. The only restriction we impose is that the boundary of the computational domain is circular so as to preserve the effectiveness of the spherical PML. As a prototypical example we consider

$$
\left\{\begin{array}{l}
\delta_{x}=0.8 x\left(1-\arctan ^{2}(4 x)\right) \cdot(1+\exp (-|x|)) \cdot\left(1-\frac{|x|}{\sqrt{R^{2}-y^{2}}}\right)^{2}, \\
\delta_{y}=\mp A \sin ^{2}\left(\pi y^{2} / R T\right) \cdot\left(1+\exp \left(\mp \rho_{i} y\right)\right) \cdot\left(1-\frac{|y|}{\sqrt{R^{2}-x^{2}}}\right)^{2},
\end{array}\right.
$$

where $A$ is the displacement amplitude, $T$ is the displacement period, and $\rho_{i}$ is the inner radius of the original mesh. For our purpose, we let $A=$ 55 and $T=15$. The visualized domain, as defined by (21), is shown in Figure 6 and numerical output can be found in Table 3(b). We anticipate that such a deformed geometry can be realized in practice by applying stress to a waveguide on top and bottom deforming the geometry into an almost rectangular shape. In Figure 7, we plot the magnitude of hybridized magnetic fields with modal order $m=4$, with $\varepsilon_{r}=\varepsilon_{r, 1}$ and $\varepsilon_{r, 2}$. The conducting interface has been stretched, which induces a stronger plasmon interaction, 


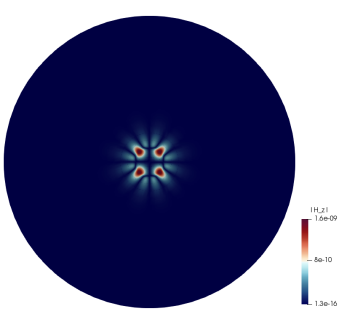

(a)

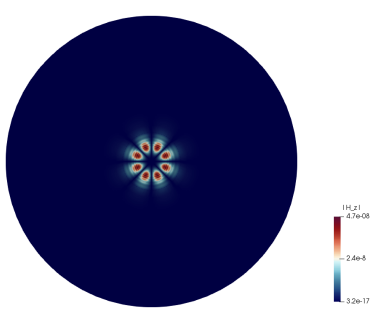

(c)

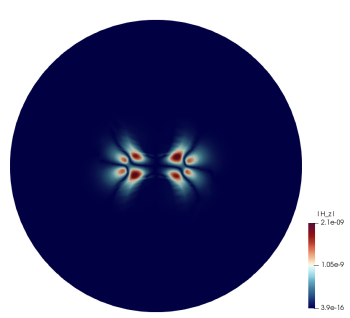

(b)

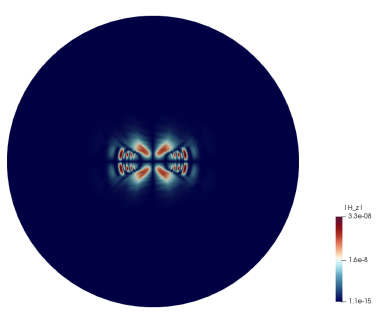

(d)

Figure 7: An example of $\left|H_{z}\right|$ eigenmode before and after mesh deformation. (a) \& (b) are obtained using $\varepsilon_{r, 1}$ with modal order of $m=4$ and (c) \& (d) via $\varepsilon_{r, 2}$ with $m=4$.

and in turn, an improved quality factor. From Table 3, we observe that the spectrum can be manipulated by changing the geometry and that the relationship between the choice of $\varepsilon_{r}$ and $\eta$ is not trivial. This presents a potential future research topic for the designing of optical devices.

\section{Conclusions and Outlook}

In this paper, we formulated a variational framework for the numerical simulation of guided modes in a waveguide setting with gradient-index host. This resulted in a quartic eigenvalue problem, which was linearized via a quadratification approach. The eigenmodes of interest are electromagnetic SPPs that arise on a conducting closed curve, e.g., graphene-coated waveguide. The interface is modeled by an idealized, oriented hypersurface.

One of the main advantages offered by our approach is a generalization of material parameters and geometric configuration. We tested our numerical treatment of the quartic eigenvalue problem with analytical predictions in the case of an isotropic medium, and observed excellent agreement. We assessed the relative strength of computed eigenmodes by quantifying eigenvalues via the quality factor, and demonstrated using concrete examples that it is 
possible to achieve a better quality factor. An improved quality factor is observed for both gradient-index waveguide and generalized geometry.

Ideally, we wish to solve the following optimization problem:

$$
\begin{array}{ll}
\underset{(\boldsymbol{x}), \mu_{r}(\boldsymbol{x}), \Sigma}{\operatorname{maximize}} & \operatorname{Re} k_{z}\left(\varepsilon_{r}(\boldsymbol{x}), \mu_{r}(\boldsymbol{x}), \Sigma\right) / \operatorname{Im} k_{z}\left(\varepsilon_{r}(\boldsymbol{x}), \mu_{r}(\boldsymbol{x}), \Sigma\right) \\
\text { subject to } & {\left[\varepsilon_{r}\right]_{\Sigma}=\left[\mu_{r}\right]_{\Sigma}=0, \text { with egularity assumptions, }} \\
& l(\Sigma)=c, \text { where } c \text { is constant. }
\end{array}
$$

Here, $l(\cdot)$ denotes the length of the curve. In the special case where $\varepsilon_{r} \equiv 1$ and $\Sigma$ is two infinite parallel layers, the optimization problem reduces to one discussed in [38. We observe that these generalized constraints can be used as a basis for solving related shape optimization problems for complicated multilayer optical devices, which is the subject of future research.

\section{Appendix A. Derivation of the weak form}

In this appendix, we carry out in detail the derivation of our weak formulation (9). As a preliminary step, we explain how the longitudinal component of the guided mode is derived.

\section{Appendix A.1. Longitudinal component}

The transverse and the longitudinal components of the rescaled timeharmonic Maxwell's equations with $e^{i k_{z} z}$ dependence are

$$
\begin{aligned}
& \left\{\begin{aligned}
i \mu_{r} \boldsymbol{H}_{s} & =\nabla_{s} \times \hat{z} E_{z}+i k_{z} \hat{z} \times \boldsymbol{E}_{s}, \\
-i \varepsilon_{r} \boldsymbol{E}_{s} & =\nabla_{s} \times \hat{z} H_{z}+i k_{z} \hat{z} \times \boldsymbol{H}_{s},
\end{aligned}\right. \\
& \left\{\begin{array}{c}
\nabla_{s} \times \boldsymbol{E}_{s}=i \mu_{r} \hat{z} H_{z}, \\
\nabla_{s} \times \boldsymbol{H}_{s}=-i \varepsilon_{r} \hat{z} E_{z} .
\end{array}\right.
\end{aligned}
$$

and the corresponding jump conditions at an interface $\Sigma$ are

$$
\left\{\begin{aligned}
{\left[\boldsymbol{\nu} \times\left(\boldsymbol{H}_{s}+\hat{z} H_{z}\right)\right]_{\Sigma} } & =\sigma_{r}^{\Sigma}\left\{\left(\boldsymbol{\nu} \times\left(\boldsymbol{E}_{s}+\hat{z} E_{z}\right)\right) \times \boldsymbol{\nu}\right\}_{\Sigma} \\
{\left[\boldsymbol{\nu} \cdot \mu_{r}\left(\boldsymbol{H}_{s}+\hat{z} H_{z}\right)\right]_{\Sigma} } & =\left[\boldsymbol{\nu} \times\left(\boldsymbol{E}_{s}+\hat{z} E_{z}\right)\right]_{\Sigma}=\left[\boldsymbol{\nu} \cdot \varepsilon_{r}\left(\boldsymbol{E}_{s}+\hat{z} E_{z}\right)\right]_{\Sigma}=0
\end{aligned}\right.
$$

where $\boldsymbol{\nu}$ is the normal vector at $\Sigma$. Equate each component of A.3 to obtain

$$
\left\{\begin{aligned}
{\left[\left(\boldsymbol{H}_{s} \cdot \boldsymbol{\tau}\right) \hat{z}\right]_{\Sigma} } & =\sigma_{r}^{\Sigma}\left\{\left(-E_{z} \boldsymbol{\tau}\right) \times \boldsymbol{\nu}\right\}_{\Sigma}=\left.\sigma_{r}^{\Sigma} E_{z} \hat{z}\right|_{\Sigma}, \\
{\left[-H_{z} \boldsymbol{\tau}\right]_{\Sigma} } & =\sigma_{r}^{\Sigma}\left\{\left(\boldsymbol{E}_{s} \cdot \boldsymbol{\tau}\right) \hat{z} \times \boldsymbol{\nu}\right\}_{\Sigma}=\left.\sigma_{r}^{\Sigma}\left(\boldsymbol{E}_{s} \cdot \boldsymbol{\tau}\right) \boldsymbol{\tau}\right|_{\Sigma} \\
{\left[\varepsilon_{r} \boldsymbol{E}_{s}\right]_{\Sigma} \cdot \boldsymbol{\nu} } & =\left[\mu_{r} \boldsymbol{H}_{s}\right]_{\Sigma} \cdot \boldsymbol{\nu}=\left[\boldsymbol{E}_{s}\right]_{\Sigma} \cdot \boldsymbol{\tau}=\left[E_{z}\right]_{\Sigma}=0 .
\end{aligned}\right.
$$


Substitute one of A.1 into the other to obtain (A.5).

$$
\left\{\begin{array}{c}
k_{s}^{2} \boldsymbol{E}_{s}=i\left(k_{z} \nabla_{s} E_{z}+\mu_{r} \nabla_{s} \times H_{z}\right), \\
k_{s}^{2} \boldsymbol{H}_{s}=i\left(k_{z} \nabla_{s} H_{z}-\varepsilon_{r} \nabla_{s} \times E_{z}\right) .
\end{array}\right.
$$

The second-order time-harmonic Maxwell's equations are

$$
\begin{gathered}
\left(\nabla_{s}+i k_{z} \hat{z}\right) \times\left(\mu_{r}^{-1}\left(\nabla_{s}+i k_{z} \hat{z}\right) \times \boldsymbol{E}\right)-\varepsilon_{r} \boldsymbol{E}=0 \\
\left(\nabla_{s}+i k_{z} \hat{z}\right) \times\left(\varepsilon_{r}^{-1}\left(\nabla_{s}+i k_{z} \hat{z}\right) \times \boldsymbol{H}\right)-\mu_{r} \boldsymbol{H}=0
\end{gathered}
$$

Equate the $z$-component of A.6 to obtain (5).

Appendix A.2. Derivation of the weak form

We multiply (6) by $k_{s}^{4}$ and distribute it in a particular manner that eases the handling of the inhomogeneities.

$$
\left\{\begin{aligned}
-k_{s}^{4}\left(\left(\nabla_{s} \frac{1}{k_{s}^{2}}\right) \cdot(\right. & \left.\left.\varepsilon_{r} \nabla_{s} E_{z}\right)+\frac{1}{k_{s}^{2}} \nabla_{s} \cdot\left(\varepsilon_{r} \nabla_{s} E_{z}\right)\right) \\
& -k_{z} k_{s}^{4}\left(\left(\nabla_{s} \frac{1}{k_{s}^{2}}\right) \cdot\left(\nabla_{s} \times \hat{z} H_{z}\right)\right)-\varepsilon_{r} k_{s}^{4} E_{z}=0 \\
-k_{s}^{4}\left(\left(\nabla_{s} \frac{1}{k_{s}^{2}}\right) \cdot(\right. & \left.\left.\mu_{r} \nabla_{s} H_{z}\right)+\frac{1}{k_{s}^{2}} \nabla_{s} \cdot\left(\mu_{r} \nabla_{s} H_{z}\right)\right) \\
& +k_{z} k_{s}^{4}\left(\left(\nabla_{s} \frac{1}{k_{s}^{2}}\right) \cdot\left(\nabla_{s} \times \hat{z} E_{z}\right)\right)-\mu_{r} k_{s}^{4} H_{z}=0
\end{aligned}\right.
$$

Since $\nabla_{s} k_{s}^{-2}=-k_{s}^{-4} \nabla_{s} k_{s}^{2}$, some algebra shows that the expression in the first line of A.7) is equivalent to

$$
\begin{aligned}
-k_{s}^{4} & \left(\left(\nabla_{s} \frac{1}{k_{s}^{2}}\right) \cdot\left(\varepsilon_{r} \nabla_{s} E_{z}\right)+\frac{1}{k_{s}^{2}} \nabla_{s} \cdot\left(\varepsilon_{r} \nabla_{s} E_{z}\right)\right) \\
& =\nabla_{s} k_{s}^{2} \cdot\left(\varepsilon_{r} \nabla_{s} E_{z}\right)-k_{s}^{2} \nabla_{s} \cdot\left(\varepsilon_{r} \nabla_{s} E_{z}\right) \\
& =\nabla_{s} \cdot\left(k_{s}^{2}\left(\varepsilon_{r} \nabla_{s} E_{z}\right)\right)-2 k_{s}^{2} \nabla_{s} \cdot\left(\varepsilon_{r} \nabla E_{z}\right) .
\end{aligned}
$$

Additionally, we note that the curl terms in A.7 can be written as

$$
\begin{aligned}
-k_{z} k_{s}^{4} & \left(\left(\nabla_{s} \frac{1}{k_{s}^{2}}\right) \cdot\left(\nabla_{s} \times \hat{z} H_{z}\right)+\frac{1}{k_{s}^{2}} \nabla_{s} \cdot\left(\nabla_{s} \times \hat{z} H_{z}\right)\right) \\
=k_{z} \nabla_{s} k_{s}^{2} \cdot\left(\nabla_{s} \times \hat{z} H_{z}\right)-k_{z} k_{s}^{2} \nabla_{s} \cdot\left(\nabla_{s} \times \hat{z} H_{z}\right) & \\
= & k_{z} \nabla_{s} \cdot\left(k_{s}^{2}\left(\nabla_{s} \times \hat{z} H_{z}\right)\right)-2 k_{z} k_{s}^{2} \nabla_{s} \cdot\left(\nabla_{s} \times \hat{z} H_{z}\right) .
\end{aligned}
$$


Even though the last term vanishes, we keep it, as it will be later utilized to express the interface contribution nicely.

We now test with smooth functions $\varphi$ and $\psi$, and integrate by parts to arrive at

$$
\begin{gathered}
-\left(k_{s}^{2} \varepsilon_{r} \nabla_{s} E_{z}, \nabla_{s} \varphi\right)+2\left(\varepsilon_{r} \nabla_{s} E_{z}, \nabla_{s}\left(\bar{k}_{s}^{2} \varphi\right)\right)-k_{z}\left(k_{s}^{2} \nabla_{s} \times \hat{z} H_{z}, \nabla_{s} \varphi\right) \\
+2 k_{z}\left(\nabla_{s} \times \hat{z} H_{z}, \nabla_{s}\left(\bar{k}_{s}^{2} \varphi\right)\right)-\left(\varepsilon_{r} k_{s}^{4} E_{z}, \varphi\right) \\
-\left(k_{s}^{2} \mu_{r} \nabla_{s} H_{z}, \nabla_{s} \psi\right)+2\left(\mu_{r} \nabla_{s} H_{z}, \nabla_{s}\left(\bar{k}_{s}^{2} \psi\right)\right)+k_{z}\left(k_{s}^{2} \nabla_{s} \times \hat{z} E_{z}, \nabla_{s} \psi\right) \\
-2 k_{z}\left(\nabla_{s} \times \hat{z} E_{z}, \nabla_{s}\left(\bar{k}_{s}^{2} \psi\right)\right)-\left(\mu_{r} k_{s}^{4} H_{z}, \psi\right) \\
+\left\langle\left[k_{s}^{2} \varepsilon_{r} \partial_{\nu} E_{z}+k_{s}^{2} k_{z} \partial_{\tau} H_{z}\right]_{\Sigma}, \varphi\right\rangle_{\Sigma}+\left\langle\left[k_{s}^{2} \mu_{r} \partial_{\nu} H_{z}-k_{s}^{2} k_{z} \partial_{\tau} E_{z}\right]_{\Sigma}, \psi\right\rangle_{\Sigma} .
\end{gathered}
$$

Using (3), (7), and A.5, the interface contributions simplify to

$$
\begin{array}{r}
\left\langle\left[k_{s}^{2}\left(\varepsilon_{r} \partial_{\nu} E_{z}+k_{z} \partial_{\tau} H_{z}\right)\right]_{\Sigma}, \varphi\right\rangle_{\Sigma}+\left\langle\left[k_{s}^{2}\left(\mu_{r} \partial_{\nu} H_{z}-k_{z} \partial_{\tau} E_{z}\right)\right]_{\Sigma}, \psi\right\rangle_{\Sigma} \\
=-i\left\langle\left[k_{s}^{4} \boldsymbol{H}_{\tau}\right]_{\Sigma}, \varphi\right\rangle_{\Sigma}-i\left\langle\left[k_{s}^{4} \boldsymbol{E}_{\tau}\right]_{\Sigma}, \psi\right\rangle_{\Sigma}=-i \sigma_{r}^{\Sigma}\left\langle k_{s}^{4} E_{z}, \varphi\right\rangle_{\Sigma}
\end{array}
$$

Expand the $\nabla_{s}\left(\bar{k}_{s}^{2} \varphi\right)$ and $\nabla_{s}\left(\bar{k}_{s}^{2} \psi\right)$ to arrive at

$$
\begin{aligned}
& \left(\mu_{r} \varepsilon_{r}^{2} \nabla_{s} E_{z}, \nabla_{s} \varphi\right)+2\left(\varepsilon_{r} \nabla_{s} E_{z}, \nabla_{s}\left(\bar{\varepsilon}_{r} \bar{\mu}_{r}\right) \varphi\right) \\
& +k_{z}\left(\mu_{r} \varepsilon_{r} \nabla_{s} \times \hat{z} H_{z}, \nabla_{s} \varphi\right)-k_{z}^{2}\left(\varepsilon_{r} \nabla_{s} E_{z}, \nabla_{s} \varphi\right) \\
& +2 k_{z}\left(\nabla_{s} \times \hat{z} H_{z}, \nabla_{s}\left(\bar{\varepsilon}_{r} \bar{\mu}_{r}\right) \varphi\right)-k_{z}^{3}\left(\nabla_{s} \times \hat{z} H_{z}, \nabla_{s} \varphi\right)-\left(\varepsilon_{r} k_{s}^{4} E_{z}, \varphi\right) \\
& +\left(\varepsilon_{r} \mu_{r}^{2} \nabla_{s} H_{z}, \nabla_{s} \psi\right)+2\left(\mu_{r} \nabla_{s} H_{z}, \nabla_{s}\left(\bar{\varepsilon}_{r} \bar{\mu}_{r}\right) \psi\right) \\
& -k_{z}\left(\mu_{r} \varepsilon_{r} \nabla_{s} \times \hat{z} E_{z}, \nabla_{s} \psi\right)-k_{z}^{2}\left(\mu_{r} \nabla_{s} H_{z}, \nabla_{s} \psi\right) \\
& -2 k_{z}\left(\nabla_{s} \times \hat{z} E_{z}, \nabla_{s}\left(\bar{\varepsilon}_{r} \bar{\mu}_{r}\right) \psi\right)+k_{z}^{3}\left(\nabla_{s} \times \hat{z} E_{z}, \nabla_{s} \psi\right)-\left(\mu_{r} k_{s}^{4} H_{z}, \psi\right) \\
& -i \sigma_{r}^{\Sigma}\left\langle k_{s}^{4} E_{z}, \varphi\right\rangle_{\Sigma}=0 \text {. }
\end{aligned}
$$

\section{References}

[1] K. S. Novoselov, S. V. M. A. K. Geim, D. Jiang, Y. Zhang, S. V. Dubonos, I. V. Grigorieva, A. A. Firsov, Electric field effect in atomically thin carbon films, Science 306 (2004) 666.

[2] Y. V. Bludov, A. Ferreira, N. M. R. Peres, M. I. Vasileskiy, A primer on surface plasmon-polaritons in graphene, International Journal of Modern Physics 27 (10) (2013) 1341001. 
[3] J. Liu, X. Zhai, L.-L. Wang, H.-J. Li, F. Xie, Q. Lin, S.-X. Xia, Analysis of mid-infrared surface plasmon modes in a graphene-based cylindrical hybrid waveguide, Plasmonics 11 (2016) 703-711.

[4] R. F. Oulton, V. J. Sorger, T. Zentgraf, R.-M. Ma, C. Gladden, L. Dai, G. Bartal, X.Zhang, Plasmon lasers at deep subwavelength scale, Nature Letters 461 (2009) 629-632.

[5] A. Vakil, N. Engheta, Transformation optics using graphene, Science 332 (2011) 1291.

[6] B. Gavin, A. Miedlar, E. Polizzi, Feast eigensolver for nonlinear eigenvalue problems, Journal of Computational Science 27 (2018) 107-117.

[7] J. Gopalakrishnan, L. Grubisic, J. Ovall, B. Q. Parker, Analysis of feast spectral approximations using the dpg discretization, Computational Methods in Applied Mathematics 19 (2019) 251-266.

[8] J. Gopalakrishnan, L. Grubisic, J. Ovall, Spectral discretization errors in filtered subspace iteration, Mathematics of Computation 89 (2020) 321.

[9] Z. Bai, D. Lu, B. Vandereycken, Robust rayleigh quotient minimization and nonlinear eigenvalue problems, Siam Journal on Scientific Computing 40 (2018) A3495-A3522.

[10] D. Arndt, W. Bangerth, T. C. Clevenger, D. Davydov, M. Fehling, D. Garcia-Sanchez, G. Harper, T. Heister, L. Heltai, M. Kronbichler, R. M. Kynch, M. Maier, J.-P. Pelteret, B. Turcksin, D. Wells, The deal.II library, version 9.1, Journal of Numerical MathematicsAccepted (2019). doi:10.1515/jnma-2019-0064.

URL https://dealii.org/deal91-preprint.pdf

[11] F. D. Terán, F. M. Dopico, D. S. Mackey, Spectral equivalence of matrix polynomials and the index sum theorem, Linear Algebra and its Applications 459 (2014).

[12] J. Xu, N. Shi, Y. Chen, X. Lu, H. Wei, Y. Lu, N. Liu, B.Zhang, J.Wang, Tm01 mode in a cylindrical hybrid plasmonic waveguide with large propagation length, Applied Optics 57 (2018) 4043-4047.

[13] Y. Gao, G. Ren, B. Zhu, H. Liu, Y. Lian, S. Jian, Analytical model for plasmon modes in graphene-coated nanowire, Optics Express 22 (2014) 24322-24331. 
[14] Y. Gao, G. Ren, B. Zhu, J. Wang, S. Jian, Single-mode graphene-coated nanowire plasmonic waveguide, Optics Letters 39 (2014) 5909-5912.

[15] Y. Xu, Y. Fu, H. Chen, Planar gradient metamaterials, Nature Reviews Materials 1 (2016) 16067.

[16] F. Moharrami, Z. Atlasbaf, Tunable grin lensing based on graphenedielectric multilayer metamaterials, Journal of Optics 22 (2020).

[17] J. C. Zhang, L. M. Xiong, M. Fang, H. B. He, Wide-angle and broadband graded-refractive-index antireflection coatings, Chinese Physics B 22 (2012) 044201.

[18] Q. I. Dai, Y. H. Lo, W. C. Chew, L. J. Jiang, An efficiently preconditioned eigenanalysis of inhomogeneously loaded rectangular cavities, IEEE Antennas Wireless Propagation Letters 12 (2013) 58-61.

[19] Q. I. Dai, W. C. Chew, L. J. Jiang, Differential forms inspired discretization for finite element analysis of inhomogeneous waveguides, Progress in Electromagnetics Research 143 (2013) 745-760.

[20] W. C. Chew, Waves and Fields in Inhomogeneous Media, Wiley-IEEE Press, New York, 1999.

[21] D. A. White, J. M. Koning, Computing solenoidal eigenmodes of the vector helmholtz equation: A novel approach, IEEE Transactions on Magnetics 38 (2002) 3420-3425.

[22] Z. Shao, W. Porod, C. S. Lent, D. J. Kirkner, An eigenvalue method for open-boundary quantum transmission problems, Journal of Applied Physics 78 (1995) 2177.

[23] E. Polizzi, Density-matrix-based algorithms for solving eigenvalue problems, Physics Review B 79 (2009) 115112.

[24] V. Hernandez, J. E. Roman, V. Vidal, A scalable and flexible toolkit for the solution of eigenvalue problems, ACM Transactions on Mathematical Software 31 (2005).

[25] J. Bezanson, A. Edelman, S. Karpinski, V. B. Shah, Julia: A Fresh Approach to Numerical Computing, SIAM Review 59 (2017) 65.

[26] M. Maier, D. Margetis, M. Luskin, Dipole excitation of surface plasmon on a conducting sheet: finite element approximation and validation, Journal of Computational Physics 339 (2017) 126-145. 
[27] Z. Drmac, I. Glibic, An algorithm for the complete solution of the quartic eigenvalue problem, Linear Algebra and its Applications 459 (2014).

[28] F. Collino, P. Monk, The perfectly matched layer in curvilinear coordinates, SIAM Journal on Scientific Computing 19 (1998) 2061-2090.

[29] P. Monk, Finite Element Methods for Maxwell's Equations, Numerical Mathematics and Scientific Computation, Oxford University Press, 2003.

[30] A. Konrad, Vector variational formulation of electromagnetic fields in anistropic media, IEEE Transactions on Microwave Theory and Techniques 24 (1976) 553.

[31] J. L. Coulomb, Finite element three dimensional magnetic field computation, IEEE Transaction on Magnetics 17 (1981) 3241.

[32] B. M. A. Rahman, J. B. Davie, Penalty function improvement of waveguide solution by finite elements, IEEE Transaction on Microwave Theory and Techniques 32 (1984) 922.

[33] J. P. Webb, Efficient generation of divergence free fields for the finite element analysis of 3D cavity resonances, IEEE Transaction on Magnetics 24 (1988) 162.

[34] A. Konrad, A direct three-dimensional finite element method for the solution of electromagnetic fields in cavities, IEEE Transaction on Magnetics 6 (1985) 2276.

[35] A. J. Kobelansky, J. P. Webb, Eliminating spurious modes in finite element waveguide problems by using divergence-free fields, Electronic Letters 22 (1986) 569.

[36] D. S. Mackey, N. Mackey, C. Mehl, V. Mehrmann, Möbius transformations of matrix polynomials, Linear Algebra and its Applications 470 (2014) 120-184.

[37] S. Balay, K. Buschelman, V. Eijkhout, W. D. Gropp, D. Kaushik, M. G. Knepley, L. C. McInnes, B. F. Smith, H. Zhang, Petsc users manual, Technical Report ANL-95/11 - Revision 2.1.5 Argonne National Laboratory (2004).

[38] J. Song, M. Maier, M. Luskin, Adaptive finite element simulations of waveguide configurations involving parallel $2 \mathrm{~d}$ material sheets, Computer Methods in Applied Mechanics and Engineering 351 (2019) 20-34. 